\title{
The Terwilliger algebra of the incidence graphs of Johnson geometry
}

\author{
Qian Kong \\ College of Engineering \\ Nanjing Agricultural University, Nanjing 210031, China \\ Sch. Math. Sci. \& Lab. Math. Com. Sys. \\ Beijing Normal University, Beijing 100875, China \\ kongqian@njau.edu.cn \\ Benjian Lv \\ Sch. Math. Sci. \& Lab. Math. Com. Sys. \\ Beijing Normal University, Beijing 100875, China \\ benjian@mail.bnu.edu.cn \\ Kaishun Wang* \\ Sch. Math. Sci. \& Lab. Math. Com. Sys. \\ Beijing Normal University, Beijing 100875, China \\ wangks@bnu . edu . cn
}

Submitted: Jan 4, 2012; Accepted: Oct 8, 2013; Published: Oct 21, 2013

Mathematics Subject Classifications: 05E30

\begin{abstract}
In 2007, Levstein and Maldonado computed the Terwilliger algebra of the Johnson graph $J(n, m)$ when $3 m \leqslant n$. It is well known that the halved graphs of the incidence graph $J(n, m, m+1)$ of Johnson geometry are Johnson graphs. In this paper, we determine the Terwilliger algebra of $J(n, m, m+1)$ when $3 m \leqslant n$, give two bases of this algebra, and calculate its dimension.
\end{abstract}

Keywords: Terwilliger algebra; Johnson graph; incidence graph; Johnson geometry

\section{Introduction}

Let $\Gamma=(X, R)$ denote a simple connected graph with the vertex set $X$ and the edge set $R$. Suppose $\operatorname{Mat}_{X}(\mathbb{C})$ denotes the algebra over the complex number field $\mathbb{C}$ consisting of

${ }^{*}$ Corresponding author 
all matrices whose rows and columns are indexed by elements of $X$. For vertices $x$ and $y$, $\partial(x, y)$ denotes the distance between $x$ and $y$, i.e., the length of a shortest path connecting $x$ and $y$. Fix a vertex $x \in X$. Let $D(x)=\max \{\partial(x, y) \mid y \in X\}$ denote the diameter with respect to $x$. For each $i \in\{0,1, \ldots, D(x)\}$, let $\Gamma_{i}(x)=\{y \in X \mid \partial(x, y)=i\}$ and define $E_{i}^{*}=E_{i}^{*}(x)$ to be the diagonal matrix in $\operatorname{Mat}_{X}(\mathbb{C})$ with $y y$-entry

$$
\left(E_{i}^{*}\right)_{y y}= \begin{cases}1, & \text { if } y \in \Gamma_{i}(x) \\ 0, & \text { otherwise }\end{cases}
$$

The subalgebra $\mathcal{T}=\mathcal{T}(x)$ of $\operatorname{Mat}_{X}(\mathbb{C})$ generated by the adjacency matrix $A$ of $\Gamma$ and $E_{0}^{*}, E_{1}^{*}, \ldots, E_{D(x)}^{*}$ is called the Terwilliger algebra of $\Gamma$ with respect to $x$. Let $\mathbb{C}^{X}$ denote the vector space over $\mathbb{C}$ consisting of all column vectors whose coordinates are indexed by $X$. A $\mathcal{T}$-module is any subspace $W$ of $\mathbb{C}^{X}$ such that $\mathcal{T} W \subseteq W$. We call a nonzero $\mathcal{T}$-module irreducible if it does not properly contain a nonzero $\mathcal{T}$-module. An irreducible $\mathcal{T}$-module $W$ is thin if $\operatorname{dim} E_{i}^{*} W \leqslant 1$ for every $i$, and the graph $\Gamma$ is said to be thin with respect to $x$ if every irreducible $\mathcal{T}(x)$-module is thin.

Terwilliger [13, 14, 15] initiated the study of the Terwilliger algebra of an association scheme, which has been an important tool in studying structures of an association scheme. For more information, see [4, 5, 6]. The Terwilliger algebras of group schemes were discussed in [1, 2]. The Terwilliger algebras of some distance-regular graphs have been determined; see [17] for strongly regular graphs, [8] for Hypercubes, [1] for Hamming graphs, [12] for Johnson graphs, [10] for odd graphs.

Let $\Omega$ be a set of cardinality $n$ and let $\left(\begin{array}{c}\Omega \\ i\end{array}\right)$ denote the collection of all $i$-subsets of $\Omega$. Suppose $m$ is a nonnegative integer with $m+1 \leqslant n$. The incidence graph $J(n, m, m+1)$ of Johnson geometry is a bipartite graph with a bipartition $\left(\begin{array}{c}\Omega \\ m\end{array}\right) \cup\left(\begin{array}{c}\Omega \\ m+1\end{array}\right)$, where $y \in\left(\begin{array}{c}\Omega \\ m\end{array}\right)$ and $z \in\left(\begin{array}{c}\Omega \\ m+1\end{array}\right)$ are adjacent if $y \subseteq z$. The graph $J(n, m, m+1)$ is distance-biregular (see [3]). It is well known that the halved graphs of $J(n, m, m+1)$ are Johnson graphs.

Levstein and Maldonado [12] determined the Terwilliger algebra of the Johnson graph $J(n, m)$ when $3 m \leqslant n$. In this paper we shall determine the Terwilliger algebra of $J(n, m, m+1)$ with respect to $x \in\left(\begin{array}{c}\Omega \\ m\end{array}\right)$ when $n \geqslant 3 m$. In Section 2, we introduce some useful identities for intersection matrices. In Section 3, the Terwilliger algebra of $J(n, m, m+1)$ is described. In Section 4, we give two bases of this algebra and compute its dimension.

\section{Intersection matrices}

In this section we shall introduce intersection matrices and some related identities.

Let $V$ be a set of cardinality $v$. The inclusion matrix $W_{i, j}(v)$ is a binary matrix whose rows and columns are indexed by elements of $\left(\begin{array}{c}V \\ i\end{array}\right)$ and $\left(\begin{array}{c}V \\ j\end{array}\right)$, respectively, with the yz-entry defined by

$$
\left(W_{i, j}(v)\right)_{y z}= \begin{cases}1, & \text { if } y \subseteq z \\ 0, & \text { otherwise. }\end{cases}
$$


Observe that

$$
W_{i, j}(v) W_{j, k}(v)=\left(\begin{array}{c}
k-i \\
j-i
\end{array}\right) W_{i, k}(v) .
$$

Let $H_{i, j}^{l}(v)$ be a binary matrix whose rows and columns are indexed by elements of $\left(\begin{array}{c}V \\ i\end{array}\right)$ and $\left(\begin{array}{c}V \\ j\end{array}\right)$, respectively, and the yz-entry is defined by

$$
\left(H_{i, j}^{l}(v)\right)_{y z}= \begin{cases}1, & \text { if }|y \cap z|=l \\ 0, & \text { otherwise }\end{cases}
$$

Define

$$
C_{i, j}^{l}(v)=\sum_{g=l}^{\min (i, j)}\left(\begin{array}{l}
g \\
l
\end{array}\right) H_{i, j}^{g}(v)
$$

In order to simply the notation, we write $W_{i, j}$ for $W_{i, j}(v)$ when $v$ is clear from context, and do the same for $H_{i, j}^{l}(v)$ and $C_{i, j}^{l}(v)$. The matrices $W_{i, j}, H_{i, j}^{l}$ and $C_{i, j}^{l}$ are intersection matrices introduced in [7].

Observe $C_{i, j}^{0}$ is the all-one matrix and

$$
C_{i, j}^{\min (i, j)}= \begin{cases}W_{j, i}^{\mathrm{T}}, & \text { if } i>j \\ W_{i, j}, & \text { otherwise. }\end{cases}
$$

Lemma 2.1 ([7]) Let $V$ be a set of cardinality $v$. Write $W_{i, j}=W_{i, j}(v)$ and $C_{i, j}^{l}=C_{i, j}^{l}(v)$. Then

$$
C_{i, j}^{l} C_{j, k}^{s}=\sum_{h=\max (0, l+s-j)}^{\min (l, s)}\left(\begin{array}{c}
v-l-s \\
j-l-s+h
\end{array}\right)\left(\begin{array}{l}
i-h \\
l-h
\end{array}\right)\left(\begin{array}{c}
k-h \\
s-h
\end{array}\right) C_{i, k}^{h} .
$$

In particular, the following hold:

(i) $W_{i, j}^{\mathrm{T}} W_{i, k}=C_{j, k}^{i}$;

(ii) $C_{i, j}^{l} W_{j, k}=\left(\begin{array}{c}k-l \\ j-l\end{array}\right) C_{i, k}^{l}$;

(iii) $W_{i, k} W_{j, k}^{\mathrm{T}}=\sum_{l=\max (0, i+j-k)}^{\min (i, j)}\left(\begin{array}{c}v-i-j \\ k-i-j+l\end{array}\right) C_{i, j}^{l}$;

(iv) $W_{i, j} C_{j, k}^{l}=\sum_{h=\max (0, l+j-i)}^{\min (l, i)}\left(\begin{array}{c}v-l-i \\ j-l-i+h\end{array}\right)\left(\begin{array}{l}k-h \\ l-h\end{array}\right) C_{i, k}^{h}$.

Fix $x \in\left(\begin{array}{l}\Omega \\ m\end{array}\right)$. We then consider the adjacency matrix $A$ of $J(n, m, m+1)$ as a block matrix with respect to the partition $\{x\} \cup \Gamma_{1}(x) \cup \cdots \cup \Gamma_{D(x)}(x)$. Let $A_{i, j}$ be the submatrix of $A$ with rows indexed by vertices of $\Gamma_{i}(x)$ and columns indexed by vertices of $\Gamma_{j}(x)$.

Lemma 2.2 Given two vertices $x, y$ of $J(n, m, m+1)$. If $x \in\left(\begin{array}{l}\Omega \\ m\end{array}\right)$, then

$$
\partial(x, y)= \begin{cases}2 i, & \text { if }|y|=m \text { and }|x \cap y|=m-i \\ 2 i+1, & \text { if }|y|=m+1 \text { and }|x \cap y|=m-i .\end{cases}
$$

In particular, $D(x)=\min (2 m+1,2 n-2 m)$. 
Proof. Immediate from [9, Lemma 2.2].

Lemma 2.3 Let $I_{\left(\begin{array}{c}v \\ k\end{array}\right)}$ be the identity matrix of size $\left(\begin{array}{l}v \\ k\end{array}\right)$. Then

$$
\begin{aligned}
& A_{i, j}=0, \quad \text { if } 0 \leqslant i \leqslant j \leqslant D(x) \text { and } i \neq j-1 ; \\
& A_{2 i, 2 i+1}=I_{\left(\begin{array}{c}
m \\
m-i
\end{array}\right)} \otimes W_{i, i+1}(n-m), \quad \text { if } 0 \leqslant i \leqslant\left\lfloor\frac{D(x)-1}{2}\right\rfloor ; \\
& A_{2 i+1,2 i+2}=W_{m-i-1, m-i}^{\mathrm{T}}(m) \otimes I_{\left(\begin{array}{c}
n-m \\
i+1
\end{array}\right)}, \quad \text { if } 0 \leqslant i \leqslant\left\lfloor\frac{D(x)}{2}\right\rfloor-1,
\end{aligned}
$$

where " $\otimes$ " denotes the Kronecker product of matrices.

Proof. (3) is directed.

Pick $y \in \Gamma_{2 i}(x), z \in \Gamma_{2 i+1}(x)$. By Lemma 2.2 we have $|y|=m,|z|=m+1$, $|x \cap y|=|x \cap z|=m-i$. Suppose $y=\alpha_{m-i} \cup \beta_{i}, z=\alpha_{m-i}^{\prime} \cup \beta_{i+1}^{\prime}$, where $\alpha_{m-i}$ and $\alpha_{m-i}^{\prime} \in\left(\begin{array}{c}x \\ m-i\end{array}\right)$, while $\beta_{i} \in\left(\begin{array}{c}\Omega \backslash x \\ i\end{array}\right)$ and $\beta_{i+1}^{\prime} \in\left(\begin{array}{c}\Omega \backslash x \\ i+1\end{array}\right)$. Then

$$
\left(A_{2 i, 2 i+1}\right)_{y z}=1 \Leftrightarrow \alpha_{m-i}=\alpha_{m-i}^{\prime} \text { and } \beta_{i} \subseteq \beta_{i+1}^{\prime} \Leftrightarrow\left(I_{\left(\begin{array}{c}
m \\
m-i
\end{array}\right)} \otimes W_{i, i+1}(n-m)\right)_{y z}=1,
$$

which leads to (4).

Similarly, 5 holds.

\section{The Terwilliger algebra}

Let $n \geqslant 3 m$ and $X$ denote the vertex set of $J(n, m, m+1)$. Fix $x \in\left(\begin{array}{c}\Omega \\ m\end{array}\right)$. In this section we shall determine the Terwilliger algebra $\mathcal{T}=\mathcal{T}(x)$ of $J(n, m, m+1)$. Hereafter the ground set of all matrices $C_{p, q}^{l}(m)$ is $x$ and that of $C_{p, q}^{l}(n-m)$ is $\Omega \backslash x$.

For $i, j \in\{0,1, \ldots, 2 m+1\}$, let $\mathcal{M}_{i, j}$ be the vector space spanned by

$$
C_{m-\left\lfloor\frac{i}{2}\right\rfloor, m-\left\lfloor\frac{j}{2}\right\rfloor}^{l}(m) \otimes C_{\left\lceil\frac{i}{2}\right\rceil,\left\lceil\frac{j}{2}\right\rceil}^{s}(n-m),
$$

where

$$
0 \leqslant l \leqslant \min \left(m-\left\lfloor\frac{i}{2}\right\rfloor, m-\left\lfloor\frac{j}{2}\right\rfloor\right), 0 \leqslant s \leqslant \min \left(\left\lceil\frac{i}{2}\right\rceil,\left\lceil\frac{j}{2}\right\rceil\right) .
$$

Write

$$
\mathcal{M}=\bigoplus_{i, j=0}^{2 m+1} L\left(\mathcal{M}_{i, j}\right)
$$

where $L\left(\mathcal{M}_{i, j}\right)=\left\{L(M) \in \operatorname{Mat}_{X}(\mathbb{C}) \mid M \in \mathcal{M}_{i, j}\right\}$, and

$$
L(M)_{\Gamma_{k}(x) \times \Gamma_{l}(x)}= \begin{cases}M, & \text { if } k=i \text { and } l=j, \\ 0, & \text { otherwise. }\end{cases}
$$

Note that $\mathcal{M}$ is a vector space. By Lemma 2.1, $\mathcal{M}$ is an algebra. In the remaining of this section we shall prove $\mathcal{T}=\mathcal{M}$. 
Lemma 3.1 The Terwilliger algebra $\mathcal{T}$ is a subalgebra of $\mathcal{M}$.

Proof. By Lemma 2.3 we have $A \in \mathcal{M}$. For $0 \leqslant i \leqslant 2 m+1$, since

$$
E_{i}^{*}=E_{i}^{*}(x)=L\left(C_{m-\left\lfloor\frac{i}{2}\right\rfloor, m-\left\lfloor\frac{i}{2}\right\rfloor}^{m-\left\lfloor\frac{i}{2}\right\rfloor}(m) \otimes C_{\left\lceil\frac{i}{2}\right\rceil,\left\lceil\frac{i}{2}\right\rceil}^{\left\lceil\frac{i}{2}\right\rceil}(n-m)\right) \in \mathcal{M},
$$

we get $\mathcal{T} \subseteq \mathcal{M}$.

For $i, j \in\{0,1, \ldots, 2 m+1\}$, let $\mathcal{T}_{i, j}=\left\{M_{i, j} \mid M \in \mathcal{T}\right\}$, where $M_{i, j}$ is the submatrix of $M$ with rows indexed by vertices of $\Gamma_{i}(x)$ and columns indexed by vertices of $\Gamma_{j}(x)$. Since $\mathcal{T}$ is an algebra, each $\mathcal{T}_{i, j}$ is a vector space. Since $\mathcal{T} E_{j}^{*} \mathcal{T} \subseteq \mathcal{T},\left(\mathcal{T} E_{j}^{*} \mathcal{T}\right)_{i, k} \subseteq \mathcal{T}_{i, k}$, which gives

$$
\mathcal{T}_{i, j} \mathcal{T}_{j, k} \subseteq \mathcal{T}_{i, k}
$$

Since $A, E_{i}^{*} \in \mathcal{T}$, we have $E_{i_{1}}^{*} A E_{i_{2}}^{*} A E_{i_{3}}^{*} \cdots A E_{i_{p-1}}^{*} A E_{i_{p}}^{*} \in E_{i_{1}}^{*} \mathcal{T} E_{i_{p}}^{*}$, from which it follows that

$$
A_{i_{1}, i_{2}} A_{i_{2}, i_{3}} \cdots A_{i_{p-2}, i_{p-1}} A_{i_{p-1}, i_{p}} \in \mathcal{T}_{i_{1}, i_{p}},
$$

where $0 \leqslant i_{s} \leqslant 2 m+1$ for any $s \in\{1, \ldots, p\}$.

Note that

$$
W_{m-\left\lfloor\frac{h}{2}\right\rfloor, m-\left\lfloor\frac{h}{2}\right\rfloor}^{\mathrm{T}}(m) \otimes W_{\left\lceil\frac{h}{2}\right\rceil,\left\lceil\frac{h}{2}\right\rceil}(n-m)=I_{\left(\begin{array}{c}
m \\
m-\left\lfloor\frac{h}{2}\right\rfloor
\end{array}\right)} \otimes I_{\left(\begin{array}{c}
n-m \\
\left\lceil\frac{h}{2}\right\rceil
\end{array}\right)} .
$$

By Lemma 2.3 and (1), for $h+1 \leqslant k$, one gets

$$
A_{h, h+1} \cdots A_{k-1, k}=\left(\left\lfloor\frac{k}{2}\right\rfloor-\left\lfloor\frac{h}{2}\right\rfloor\right) !\left(\left\lceil\frac{k}{2}\right\rceil-\left\lceil\frac{h}{2}\right\rceil\right) ! W_{m-\left\lfloor\frac{k}{2}\right\rfloor, m-\left\lfloor\frac{h}{2}\right\rfloor}^{\mathrm{T}}(m) \otimes W_{\left\lceil\frac{h}{2}\right\rceil,\left\lceil\frac{k}{2}\right\rceil}(n-m) .
$$

Hence, by (8), for $h \leqslant k$, we have

$$
W_{m-\left\lfloor\frac{k}{2}\right\rfloor, m-\left\lfloor\frac{h}{2}\right\rfloor}^{\mathrm{T}}(m) \otimes W_{\left\lceil\frac{h}{2}\right\rceil,\left\lceil\frac{k}{2}\right\rceil}(n-m) \in \mathcal{T}_{h, k} .
$$

Lemma 3.2 For $2 i+2 \leqslant j \leqslant 2 m+1$ and $0 \leqslant s \leqslant i+1$, we have

$$
C_{m-i-1, m-\left\lfloor\frac{j}{2}\right\rfloor}^{m-\left\lfloor\frac{j}{2}\right\rfloor}(m) \otimes C_{i+1,\left\lceil\frac{j}{2}\right\rceil}^{s}(n-m) \in \mathcal{T}_{2 i+2, j} .
$$

Proof. We use induction on $s$ ( $s$ decreasing from $i+1$ to 0$)$. Since

$$
C_{m-i-1, m-\left\lfloor\frac{j}{2}\right\rfloor}^{m-\left\lfloor\frac{j}{2}\right\rfloor}(m) \otimes C_{i+1,\left\lceil\frac{j}{2}\right\rceil}^{i+1}(n-m)=W_{m-\left\lfloor\frac{j}{2}\right\rfloor, m-i-1}^{\mathrm{T}}(m) \otimes W_{i+1,\left\lceil\frac{j}{2}\right\rceil}(n-m),
$$

by (9), 10) holds for $2 i+2 \leqslant j \leqslant 2 m+1$ and $s=i+1$.

Assume that $C_{m-i-1, m-\left\lfloor\frac{j}{2}\right\rfloor}^{m-\left\lfloor\frac{j}{2}\right\rfloor}(m) \otimes C_{i+1,\left\lceil\frac{j}{2}\right\rceil}^{s}(n-m) \in \mathcal{T}_{2 i+2, j}$. By $(7)$ and $(8)$ we obtain

$$
\begin{aligned}
& \left(C_{m-i-1, m-\left\lfloor\frac{j}{2}\right\rfloor}^{m-\left\lfloor\frac{j}{2}\right\rfloor}(m) \otimes C_{i+1,\left\lceil\frac{j}{2}\right\rceil}^{s}(n-m)\right)\left(A_{j, j+1} A_{j+1, j}\right) \in \mathcal{T}_{2 i+2, j} \mathcal{T}_{j, j} \subseteq \mathcal{T}_{2 i+2, j} \\
& \left(C_{m-i-1, m-\left\lfloor\frac{j}{2}\right\rfloor}^{m-\left\lfloor\frac{j}{2}\right\rfloor}(m) \otimes C_{i+1,\left\lceil\frac{j}{2}\right\rceil}^{s}(n-m)\right)\left(A_{j, j-1} A_{j-1, j}\right) \in \mathcal{T}_{2 i+2, j} \mathcal{T}_{j, j} \subseteq \mathcal{T}_{2 i+2, j}
\end{aligned}
$$


When $j$ is even, by Lemma 2.3, Lemma 2.1, (11) leads to

$$
a C_{m-i-1, m-\frac{j}{2}}^{m-\frac{j}{2}}(m) \otimes C_{i+1, \frac{j}{2}}^{s}(n-m)+b C_{m-i-1, m-\frac{j}{2}}^{m-\frac{j}{2}}(m) \otimes C_{i+1, \frac{j}{2}}^{s-1}(n-m) \in \mathcal{T}_{2 i+2, j},
$$

where $a=\left(n-m-s-\frac{j}{2}\right)\left(\frac{j}{2}-s+1\right)$ and $b=(i-s+2)\left(\frac{j}{2}-s+1\right)$. Similarly when $j$ is odd, (12) yields that

$$
a^{\prime} C_{m-i-1, m-\left\lfloor\frac{j}{2}\right\rfloor}^{m-\left\lfloor\frac{j}{2}\right\rfloor}(m) \otimes C_{i+1,\left\lceil\frac{j}{2}\right\rceil}^{s}(n-m)+b^{\prime} C_{m-i-1, m-\left\lfloor\frac{j}{2}\right\rfloor}^{m-\left\lfloor\frac{j}{2}\right\rfloor}(m) \otimes C_{i+1,\left\lceil\frac{j}{2}\right\rceil}^{s-1}(n-m)
$$

belongs to $\mathcal{T}_{2 i+2, j}$, where $a^{\prime}=\left(n-m-s-\left\lceil\frac{j}{2}\right\rceil+1\right)\left(\left\lceil\frac{j}{2}\right\rceil-s\right)$ and $b^{\prime}=(i-s+2)\left(\left\lceil\frac{j}{2}\right\rceil-s+1\right)$. Since $s \leqslant i+1 \leqslant\left\lceil\frac{j}{2}\right\rceil, b \neq 0$ and $b^{\prime} \neq 0$. Thus we have $C_{m-i-1, m-\left\lfloor\frac{j}{2}\right\rfloor}^{m-\left\lfloor\frac{j}{2}\right\rfloor}(m) \otimes C_{i+1,\left\lceil\frac{j}{2}\right\rceil}^{s-1}(n-m) \in$ $\mathcal{T}_{2 i+2, j}$.

Hence the desired result follows.

Lemma 3.3 The algebra $\mathcal{M}$ is a subalgebra of $\mathcal{T}$.

Proof. During this proof we will omit the symbol $(m)$ from matrices in front of " $\otimes$ ", and omit $(n-m)$ from matrices behind " $\otimes$ ".

In order to get the desired conclusion, we only need to show that $\mathcal{M}_{i, j} \subseteq \mathcal{T}_{i, j}$ for $i, j \in\{0,1, \ldots, 2 m+1\}$. Write $\mathcal{M}_{i, j}^{\mathrm{T}}=\left\{M^{\mathrm{T}} \mid M \in \mathcal{M}_{i, j}\right\}$ and $\mathcal{T}_{i, j}^{\mathrm{T}}=\left\{M^{\mathrm{T}} \mid M \in \mathcal{T}_{i, j}\right\}$. Since $\mathcal{M}_{j, i}=\mathcal{M}_{i, j}^{\mathrm{T}}$ and $\mathcal{T}_{j, i}=\mathcal{T}_{i, j}^{\mathrm{T}}$, it suffices to prove $\mathcal{M}_{i, j} \subseteq \mathcal{T}_{i, j}$ for $i \leqslant j$. We use induction on $i$.

Step 1. We show that $\mathcal{M}_{0, j} \subseteq \mathcal{T}_{0, j}$ for $0 \leqslant j \leqslant 2 m+1$.

According to $(6)$, the subspace $\mathcal{M}_{0, j}$ is spanned by $C_{m, m-\left\lfloor\frac{j}{2}\right\rfloor}^{l} \otimes C_{0,\left\lceil\frac{j}{2}\right\rceil}^{0}$, where $0 \leqslant l \leqslant$ $m-\left\lfloor\frac{j}{2}\right\rfloor$. Since

$$
C_{m, m-\left\lfloor\frac{j}{2}\right\rfloor}^{l} \otimes C_{0,\left\lceil\frac{j}{2}\right\rceil}^{0}=\left(\begin{array}{c}
m-\left\lfloor\frac{j}{2}\right\rfloor \\
l
\end{array}\right) W_{m-\left\lfloor\frac{j}{2}\right\rfloor, m}^{\mathrm{T}} \otimes W_{0,\left\lceil\frac{j}{2}\right\rceil}
$$

for any $l \in\left\{0,1, \ldots, m-\left\lfloor\frac{j}{2}\right\rfloor\right\}$, we get $\mathcal{M}_{0, j} \subseteq \mathcal{T}_{0, j}$ from $(9)$.

Step 2. Assume that $\mathcal{M}_{p, j} \subseteq \mathcal{T}_{p, j}$ for $p \leqslant 2 i$. We will show that $\mathcal{M}_{2 i+1, j} \subseteq \mathcal{T}_{2 i+1, j}$ and $\mathcal{M}_{2 i+2, j} \subseteq \mathcal{T}_{2 i+2, j}$.

Step 2.1. We show that $\mathcal{M}_{2 i+1, j} \subseteq \mathcal{T}_{2 i+1, j}$ for $2 i+1 \leqslant j \leqslant 2 m+1$.

It suffices to prove

$$
C_{m-i, m-\left\lfloor\frac{j}{2}\right\rfloor}^{l} \otimes C_{i+1,\left\lceil\frac{j}{2}\right\rceil}^{s} \in \mathcal{T}_{2 i+1, j}
$$

where $0 \leqslant l \leqslant m-\left\lfloor\frac{j}{2}\right\rfloor, 0 \leqslant s \leqslant i+1$.

By induction hypothesis,

$$
C_{m-i, m-\left\lfloor\frac{j}{2}\right\rfloor}^{l} \otimes C_{i,\left\lceil\frac{j}{2}\right\rceil}^{s} \in \mathcal{M}_{2 i, j} \subseteq \mathcal{T}_{2 i, j},
$$


for $0 \leqslant l \leqslant m-\left\lfloor\frac{j}{2}\right\rfloor, 0 \leqslant s \leqslant i$. Since

$$
A_{2 i, 2 i+1}^{\mathrm{T}}=I_{\left(\begin{array}{c}
m \\
m-i
\end{array}\right)} \otimes W_{i, i+1}^{\mathrm{T}} \in \mathcal{M}_{2 i, 2 i+1}^{\mathrm{T}} \subseteq \mathcal{T}_{2 i, 2 i+1}^{\mathrm{T}},
$$

we have

$$
\left(I_{\left(\begin{array}{c}
m \\
m-i
\end{array}\right)} \otimes W_{i, i+1}^{\mathrm{T}}\right)\left(C_{m-i, m-\left\lfloor\frac{j}{2}\right\rfloor}^{l} \otimes C_{i,\left\lceil\frac{j}{2}\right\rceil}^{s}\right) \in \mathcal{T}_{2 i, 2 i+1}^{\mathrm{T}} \mathcal{T}_{2 i, j} \subseteq \mathcal{T}_{2 i+1, j} .
$$

By Lemma 2.1, (13) holds for $0 \leqslant l \leqslant m-\left\lfloor\frac{j}{2}\right\rfloor$ and $0 \leqslant s \leqslant i$.

Next we shall show that $(13)$ holds for $0 \leqslant l \leqslant m-\left\lfloor\frac{j}{2}\right\rfloor$ and $s=i+1$.

By (9), for $j \leqslant k \leqslant 2 m+1$,

$$
\left(W_{m-\left\lfloor\frac{j}{2}\right\rfloor, m-i}^{\mathrm{T}} \otimes W_{i+1,\left\lceil\frac{j}{2}\right\rceil}\right)\left(W_{m-\left\lfloor\frac{k}{2}\right\rfloor, m-\left\lfloor\frac{j}{2}\right\rfloor}^{\mathrm{T}} \otimes W_{\left\lceil\frac{j}{2}\right\rceil,\left\lceil\frac{k}{2}\right\rceil}\right)\left(W_{m-\left\lfloor\frac{k}{2}\right\rfloor, m-\left\lfloor\frac{j}{2}\right\rfloor} \otimes W_{\left\lceil\frac{j}{2}\right\rceil,\left\lceil\frac{k}{2}\right\rceil}^{\mathrm{T}}\right)
$$

belongs to $\mathcal{T}_{2 i+1, j}$. By Lemma 2.1 .

$$
a C_{m-i, m-\left\lfloor\frac{j}{2}\right\rfloor}^{m-\left\lfloor\frac{k}{2}\right\rfloor} \otimes\left(\sum_{h=\max \left(0, i+1+\left\lceil\frac{j}{2}\right\rceil-\left\lceil\frac{k}{2}\right\rceil\right)}^{i+1}\left(\begin{array}{c}
n-m-i-1-\left\lceil\frac{j}{2}\right\rceil \\
\left\lceil\frac{k}{2}\right\rceil-i-1-\left\lceil\frac{j}{2}\right\rceil+h
\end{array}\right) C_{i+1,\left\lceil\frac{j}{2}\right\rceil}^{h}\right)
$$

belongs to $\mathcal{T}_{2 i+1, j}$, where $a=\left(\begin{array}{c}\left\lfloor\frac{k}{2}\right\rfloor-i \\ \left\lfloor\frac{k}{2}\right\rfloor-\left\lfloor\frac{j}{2}\right\rfloor\end{array}\right)\left(\begin{array}{c}\left\lceil\frac{k}{2}\right\rceil-i-1 \\ \left\lceil\frac{j}{2}\right\rceil-i-1\end{array}\right) \neq 0$. Since 13 holds for $0 \leqslant l \leqslant m-\left\lfloor\frac{j}{2}\right\rfloor$ and $0 \leqslant s \leqslant i$, one has

$$
\left(\begin{array}{c}
n-m-i-1-\left\lceil\frac{j}{2}\right\rceil \\
\left\lceil\frac{k}{2}\right\rceil-\left\lceil\frac{j}{2}\right\rceil
\end{array}\right) C_{m-i, m-\left\lfloor\frac{j}{2}\right\rfloor}^{m-\left\lfloor\frac{k}{2}\right\rfloor} \otimes C_{i+1,\left\lceil\frac{j}{2}\right\rceil}^{i+1} \in \mathcal{T}_{2 i+1, j} .
$$

Since $0 \leqslant 2 i+1 \leqslant j \leqslant k-1 \leqslant 2 m-1$ and $n \geqslant 3 m$, we get

$$
n-m-i-1-\left\lceil\frac{j}{2}\right\rceil \geqslant n-m-m-\left\lceil\frac{j}{2}\right\rceil \geqslant m-\left\lceil\frac{j}{2}\right\rceil \geqslant\left\lceil\frac{k}{2}\right\rceil-\left\lceil\frac{j}{2}\right\rceil \geqslant 0,
$$

and so $\left(\begin{array}{c}n-m-i-1-\left\lceil\frac{j}{2}\right\rceil \\ \left\lceil\frac{k}{2}\right\rceil-\left\lceil\frac{j}{2}\right\rceil\end{array}\right) \neq 0$. Hence 13 holds for $0 \leqslant l \leqslant m-\left\lfloor\frac{j}{2}\right\rfloor$ and $s=i+1$.

Step 2.2. We show that $\mathcal{M}_{2 i+2, j} \subseteq \mathcal{T}_{2 i+2, j}$ for $2 i+2 \leqslant j \leqslant 2 m+1$.

It suffices to prove

$$
C_{m-i-1, m-\left\lfloor\frac{j}{2}\right\rfloor}^{l} \otimes C_{i+1,\left\lceil\frac{j}{2}\right\rceil}^{s} \in \mathcal{T}_{2 i+2, j}, \quad 0 \leqslant l \leqslant m-\left\lfloor\frac{j}{2}\right\rfloor, 0 \leqslant s \leqslant i+1 .
$$

By the inductive assumption, for $0 \leqslant l \leqslant m-\left\lfloor\frac{j}{2}\right\rfloor$ and $0 \leqslant s \leqslant i+1$,

$$
C_{m-i, m-\left\lfloor\frac{j}{2}\right\rfloor}^{l} \otimes C_{i+1,\left\lceil\frac{j}{2}\right\rceil}^{s} \in \mathcal{M}_{2 i+1, j} \subseteq \mathcal{T}_{2 i+1, j}
$$

Since

$$
A_{2 i+1,2 i+2}^{\mathrm{T}}=W_{m-i-1, m-i} \otimes I_{\left(\begin{array}{c}
n-m \\
i+1
\end{array}\right)} \in \mathcal{T}_{2 i+1,2 i+2}^{\mathrm{T}}
$$


by (7) we have

$$
\left(W_{m-i-1, m-i} \otimes I_{\left(\begin{array}{c}
n-m \\
i+1
\end{array}\right)}\right)\left(C_{m-i, m-\left\lfloor\frac{j}{2}\right\rfloor}^{l} \otimes C_{i+1,\left\lceil\frac{j}{2}\right\rceil}^{s}\right) \in \mathcal{T}_{2 i+1,2 i+2}^{\mathrm{T}} \mathcal{T}_{2 i+1, j} \subseteq \mathcal{T}_{2 i+2, j}
$$

By Lemma 2.1,

$$
W_{m-i-1, m-i} C_{m-i, m-\left\lfloor\frac{j}{2}\right\rfloor}^{l}=(i+1-l) C_{m-i-1, m-\left\lfloor\frac{j}{2}\right\rfloor}^{l}+\left(m-\left\lfloor\frac{j}{2}\right\rfloor-l+1\right) C_{m-i-1, m-\left\lfloor\frac{j}{2}\right\rfloor}^{l-1} .
$$

Thus (16) implies that

$$
\left((i+1-l) C_{m-i-1, m-\left\lfloor\frac{j}{2}\right\rfloor}^{l}+\left(m-\left\lfloor\frac{j}{2}\right\rfloor-l+1\right) C_{m-i-1, m-\left\lfloor\frac{j}{2}\right\rfloor}^{l-1}\right) \otimes C_{i+1,\left\lceil\frac{j}{2}\right\rceil}^{s}
$$

belongs to $\mathcal{T}_{2 i+2, j}$, where $0 \leqslant l \leqslant m-\left\lfloor\frac{j}{2}\right\rfloor, 0 \leqslant s \leqslant i+1$. Since the coefficient of $C_{m-i-1, m-\left\lfloor\frac{j}{2}\right\rfloor}^{l-1} \otimes C_{i+1,\left\lceil\frac{j}{2}\right\rceil}^{s}$ in 17 is $m-\left\lfloor\frac{j}{2}\right\rfloor-l+1 \neq 0$, by Lemma 3.2 we get 15 .

Hence the desired result follows.

Theorem 3.4 Fix $x \in\left(\begin{array}{c}\Omega \\ m\end{array}\right)$. Let $\mathcal{T}$ be the Terwilliger algebra of $J(n, m, m+1)$ with respect to $x$ and $\mathcal{M}$ be the algebra defined in (6). If $n \geqslant 3 m$, then $\mathcal{T}=\mathcal{M}$.

Proof. Combining Lemmas 3.1 and 3.3, the desired result follows.

The condition $n \geqslant 3 m$ guarantees the coefficient of $C_{m-i, m-\left\lfloor\frac{j}{2}\right\rfloor}^{m-\left\lfloor\frac{k}{2}\right\rfloor} \otimes C_{i+1,\left\lceil\frac{j}{2}\right\rceil}^{i+1}$ in 14 is non-zero. It seems to be interesting to determine the Terwilliger algebra of $J(n, m, m+1)$ without this assumption.

Theorem 3.5 ([16, Theorem 13]) Let $\Gamma=(X, R)$ be a graph and $\mathcal{T}$ be the Terwilliger algebra of $\Gamma$ with respect to a vertex $x$. If $E_{i}^{*} \mathcal{T} E_{i}^{*}$ is symmetric for any $i \in\{0,1, \ldots, D(x)\}$, then $\Gamma$ is thin with respect to $x$.

Corollary 3.6 With reference to Theorem 3.4, $J(n, m, m+1)$ is thin with respect to $x$.

Proof. By Theorem 3.4 for any $i \in\{0,1, \ldots, D(x)\}$, the subspace $E_{i}^{*} \mathcal{T} E_{i}^{*}$ is spanned by

$$
L\left(C_{m-\left\lfloor\frac{i}{2}\right\rfloor, m-\left\lfloor\frac{i}{2}\right\rfloor}^{l}(m) \otimes C_{\left\lceil\frac{i}{2}\right\rceil,\left\lceil\frac{i}{2}\right\rceil}^{s}(n-m)\right),
$$

where $0 \leqslant l \leqslant m-\left\lfloor\frac{i}{2}\right\rfloor, 0 \leqslant s \leqslant\left\lceil\frac{i}{2}\right\rceil$. Since each element of $E_{i}^{*} \mathcal{T} E_{i}^{*}$ is symmetric, we get the conclusion from Theorem 3.5 . 


\section{Two bases of the Terwilliger algebra}

In this section we shall determine two bases of the Terwilliger algebra $\mathcal{T}$ in Theorem 3.4 . Set

$$
G_{i, j}=\left\{g \mid H_{m-\left\lfloor\frac{i}{2}\right\rfloor, m-\left\lfloor\frac{j}{2}\right\rfloor}^{g}(m) \neq 0\right\}, R_{i, j}=\left\{r \mid H_{\left\lceil\frac{i}{2}\right\rceil,\left\lceil\frac{j}{2}\right\rceil}^{r}(n-m) \neq 0\right\} .
$$

Theorem 4.1 Let $\mathcal{T}$ be as in Theorem 3.4. Then

$$
\left\{L\left(H_{m-\left\lfloor\frac{i}{2}\right\rfloor, m-\left\lfloor\frac{j}{2}\right\rfloor}^{g}(m) \otimes H_{\left\lceil\frac{i}{2}\right\rceil,\left\lceil\frac{j}{2}\right\rceil}^{r}(n-m)\right), g \in G_{i, j}, r \in R_{i, j}\right\}_{i, j=0}^{2 m+1}
$$

as well as

$$
\left\{L\left(C_{m-\left\lfloor\frac{i}{2}\right\rfloor, m-\left\lfloor\frac{j}{2}\right\rfloor}^{l}(m) \otimes C_{\left\lceil\frac{i}{2}\right\rceil,\left\lceil\frac{j}{2}\right\rceil}^{s}(n-m)\right), l \in G_{i, j}, s \in R_{i, j}\right\}_{i, j=0}^{2 m+1}
$$

are two bases of $\mathcal{T}$.

Proof. Without loss of generality, suppose $i \leqslant j$. We have $H_{i, j}^{l}(v) \neq 0$ if and only if $\max (0, i+j-v) \leqslant l \leqslant \min (i, j)$, so $\left\lceil\frac{i}{2}\right\rceil-\left|R_{i, j}\right|+1 \leqslant r \leqslant\left\lceil\frac{i}{2}\right\rceil$ when $r \in R_{i, j}$. By (2) we obtain

$$
C_{\left\lceil\frac{i}{2}\right\rceil,\left\lceil\frac{j}{2}\right\rceil}^{r}(n-m)=\sum_{h=r}^{\left\lceil\frac{i}{2}\right\rceil}\left(\begin{array}{l}
h \\
r
\end{array}\right) H_{\left\lceil\frac{i}{2}\right\rceil,\left\lceil\frac{j}{2}\right\rceil}^{h}(n-m),
$$

which implies that $H_{\left\lceil\frac{i}{2}\right\rceil,\left\lceil\frac{j}{2}\right\rceil}^{r}(n-m)$ is a linear combination of $\left\{C_{\left\lceil\frac{i}{2}\right\rceil,\left\lceil\frac{j}{2}\right\rceil}^{s}(n-m)\right\}_{s \in R_{i, j}}$ for any $r \in R_{i, j}$. Similarly, $H_{m-\left\lfloor\frac{i}{2}\right\rfloor, m-\left\lfloor\frac{j}{2}\right\rfloor}^{g}(m)$ can be expressed as a linear combination of $\left\{C_{m-\left\lfloor\frac{i}{2}\right\rfloor, m-\left\lfloor\frac{j}{2}\right\rfloor}^{l}(m)\right\}_{l \in G_{i, j}}$ for any $g \in G_{i, j}$. Hence every element of

$$
\left\{H_{m-\left\lfloor\frac{i}{2}\right\rfloor, m-\left\lfloor\frac{j}{2}\right\rfloor}^{g}(m) \otimes H_{\left\lceil\frac{i}{2}\right\rceil,\left\lceil\frac{j}{2}\right\rceil}^{r}(n-m)\right\}_{g \in G_{i, j}, r \in R_{i, j}}
$$

belongs to $\mathcal{M}_{i, j}$. Again by $(2)$, for $0 \leqslant l \leqslant m-\left\lfloor\frac{j}{2}\right\rfloor$ and $0 \leqslant s \leqslant\left\lceil\frac{i}{2}\right\rceil$,

$$
\begin{aligned}
& C_{m-\left\lfloor\frac{i}{2}\right\rfloor, m-\left\lfloor\frac{j}{2}\right\rfloor}^{l}(m) \otimes C_{\left\lceil\frac{i}{2}\right\rceil,\left\lceil\frac{j}{2}\right\rceil}^{s}(n-m) \\
= & \left(\sum_{g=l}^{m-\left\lfloor\frac{j}{2}\right\rfloor}\left(\begin{array}{l}
g \\
l
\end{array}\right) H_{m-\left\lfloor\frac{i}{2}\right\rfloor, m-\left\lfloor\frac{j}{2}\right\rfloor}^{g}(m)\right) \otimes\left(\sum_{r=s}^{\left\lceil\left\lceil\frac{i}{2}\right\rceil\right.}\left(\begin{array}{l}
r \\
s
\end{array}\right) H_{\left\lceil\frac{i}{2}\right\rceil,\left\lceil\frac{j}{2}\right\rceil}^{r}(n-m)\right) .
\end{aligned}
$$

Observe that (21) are linearly independent, so (21) is a basis of $\mathcal{M}_{i, j}$. Therefore (18) is a basis of $\mathcal{T}$.

Furthermore, by $(20)$ we get $\left\{C_{m-\left\lfloor\frac{i}{2}\right\rfloor, m-\left\lfloor\frac{j}{2}\right\rfloor}^{l}(m) \otimes C_{\left\lceil\frac{i}{2}\right\rceil,\left\lceil\frac{j}{2}\right\rceil}^{s}(n-m)\right\}_{l \in G_{i, j}, s \in R_{i, j}}$ is also a basis of $\mathcal{M}_{i, j}$, from which it follows that 190 is a basis of $\mathcal{T}$. 
Corollary 4.2 With reference to Theorem 3.4 we get the dimension of $\mathcal{T}$ is

$$
\operatorname{dim} \mathcal{T}= \begin{cases}\frac{1}{12}(m+1)(m+2)(m+3)(3 m+10)-4, & \text { if } n=3 m, \\ \frac{1}{12}(m+1)(m+2)(m+3)(3 m+10)-1, & \text { if } n=3 m+1, \\ \frac{1}{12}(m+1)(m+2)(m+3)(3 m+10), & \text { if } n \geqslant 3 m+2 .\end{cases}
$$

Proof. By Theorem 4.1,

$$
\begin{aligned}
\operatorname{dim} \mathcal{T}= & \sum_{i, j=0}^{2 m+1}\left|G_{i, j}\right|\left|R_{i, j}\right| \\
= & \sum_{i, j=0}^{2 m+1}\left(\min \left(m-\left\lfloor\frac{i}{2}\right\rfloor, m-\left\lfloor\frac{j}{2}\right\rfloor\right)-\max \left(0, m-\left\lfloor\frac{i}{2}\right\rfloor-\left\lfloor\frac{j}{2}\right\rfloor\right)+1\right) \\
& \times\left(\min \left(\left\lceil\frac{i}{2}\right\rceil,\left\lceil\frac{j}{2}\right\rceil\right)-\max \left(0,\left\lceil\frac{i}{2}\right\rceil+\left\lceil\frac{j}{2}\right\rceil-n+m\right)+1\right) .
\end{aligned}
$$

By zigzag calculation, we get the desired result.

\section{Concluding Remark}

We conclude this paper with the following remarks:

(i) Let $\Omega$ be a set of cardinality $n$ and let $J(n, m)$ be the Johnson graph based on $\Omega$ with $n \geqslant 3 m$. Fix an $m$-subset $x$ of $\Omega$. Let $\mathcal{T}^{\prime}=\mathcal{T}^{\prime}(x)$ and $\mathcal{T}=\mathcal{T}(x)$ be the Terwilliger algebra of $J(n, m)$ and $J(n, m, m+1)$ with respect to $x$, respectively. Since $\bigoplus_{i, j=0}^{m} E_{2 i}^{*}(x) \mathcal{T} E_{2 j}^{*}(x)$ is an algebra, $\left\{L\left(H_{m-i, m-j}^{g}(m) \otimes H_{i, j}^{r}(n-m)\right), g \in G_{2 i, 2 j}, r \in R_{2 i, 2 j}\right\}_{i, j=0}^{m}$ is a basis of $\bigoplus_{i, j=0}^{m} E_{2 i}^{*}(x) \mathcal{T} E_{2 j}^{*}(x)$ by Theorem 4.1. By [12, Definition 4.2, Lemma 4.4, Theorem 5.9] this basis coincides with that of $\mathcal{T}^{\prime}$, which implies that $\mathcal{T}^{\prime} \simeq \bigoplus_{i, j=0}^{m} E_{2 i}^{*}(x) \mathcal{T} E_{2 j}^{*}(x)$.

(ii) Using the same method, the Terwilliger algebra of $J(n, m, m+1)$ with respect to an $(m+1)$-subset may be determined.

\section{Acknowledgement}

We are indebted to the anonymous reviewer for his detailed reports. We would like to thank Professor Hiroshi Suzuki for proposing this problem. This research is supported by NSFC $(11301270,11271047)$.

\section{References}

[1] P. Balmaceda, M. Oura, The Terwilliger algebras of the group association schemes of $S_{5}$ and $A_{5}$, Kyushu J. Math. 48 (1994) 221-231.

[2] E. Bannai, A. Munemasa, The Terwilliger algebras of group association schemes, Kyushu J. Math. 49 (1995) 93-102. 
[3] A.E. Brouwer, A.M. Cohen, A. Neumaier, Distance-Regular Graphs, Springer-Verlag, Berlin, Heidelberg, 1989.

[4] J.S. Caughman IV, The Terwilliger algebra for bipartite $P$ - and $Q$-polynomial association schemes, Discrete Math. 196 (1999) 65-95.

[5] J.S. Caughman, M.S. MacLean, P. Terwilliger, The Terwilliger algebra of an almostbipartite $P$ - and $Q$-polynomial association scheme, Discrete Math. 292 (2005) 17-44.

[6] B. Curtin, The Terwilliger algebra of a 2-homogeneous bipartite distance-regular graph, J. Combin. Theory Ser. A 81 (2001) 125-141.

[7] N. Ghareghani, E. Ghorbani, M. Mohammad-Noori, Intersection matrices revisited, J. Combin. Des. 20 (2012) 383-397.

[8] J. Go, The Terwilliger algebra of the hypercube, European J. Combin. 23 (2002) 399-429.

[9] A. Hiraki, A characterization of the doubled Grassmann graphs, the doubled Odd graphs, and the Odd graphs by strongly closed subgraphs, European J. Combin. 24 (2003) 161-171.

[10] Q. Kong, B. Lv, K. Wang, The Terwilliger algebra of odd graphs, Discrete Math. 313 (2013) 698-703.

[11] F. Levstein, C. Maldonado, D. Penazzi, The Terwilliger algebra of a Hamming scheme $H(d, q)$, European J. Combin. 27 (2006) 1-10.

[12] F. Levstein, C. Maldonado, The Terwilliger algebra of the Johnson schemes, Discrete Math. 307 (2007) 1621-1635.

[13] P. Terwilliger, The subconstituent algebra of an association scheme I, J. Algebr. Combin. 1 (1992) 363-388.

[14] P. Terwilliger, The subconstituent algebra of an association scheme II, J. Algebr. Combin. 2 (1993) 73-103.

[15] P. Terwilliger, The subconstituent algebra of an association scheme III, J. Algebr. Combin. 2 (1993) 177-210.

[16] P. Terwilliger, Algebraic Graph Theory, Hand-Written Lecture Note of Paul Terwilliger, Rewritten and Added Comments by H. Suzuki, http://subsite.icu.ac. jp/people/hsuzuki/lecturenote

[17] M. Tomiyama, N. Yamazaki, The subconstituent algebra of a strongly regular graph, Kyushu J. Math. 48 (1994) 323-334. 